\title{
Avaliação do risco de suicídio: Um estudo entre universitários da área da saúde
}

\author{
Suicide risk assessment: A study among university students in the health area \\ Evaluación del riesgo de suicidio: Un estudio entre estudiantes universitarios de la salud
}

Recebido: 06/07/2021 | Revisado: 10/07/2021 | Aceito: 14/07/2021 | Publicado: 24/07/2021

Ana Patrícia Fonseca Coelho Galvão
ORCID: https://orcid.org/0000-0003-3376-5678
Faculdade de Ciências Médicas da Santa Casa de São Paulo, Brasil
E-mail: apfcoelho@gmail.com
Geovanna Carvalho Caldas Vilar de Lima
ORCID: https://orcid.org/0000-0002-1245-1138
Universidade Ceuma, Brasil
E-mail:geovannavilar@hotmail.com
Francisca Bruna Arruda Aragão
ORCID: https://orcid.org/0000-0002-1191-0988
Universidade de São Paulo, Brasil
E-mail: aragao_bruna@usp.br
Ricardo Riyoiti Uchida
Faculdade de Ciências Médicas da Santa de São Paulo, Brasil
E-mail: rruchida@uol.com.br

\section{Resumo}

O ingresso na universidade representa fase de mudanças no convívio social e nas atividades cotidianas com autonomia e liberdade ao estudante, e implica em responsabilidades frente às cobranças acadêmicas. Percebemos que, em decorrência desses fatores, muitas situações podem tornar o estudante vulnerável aos transtornos mentais. O objetivo do estudo foi avaliar o perfil de saúde mental com relação ao risco suicida entre estudantes universitários de uma instituição de ensino superior. Trata-se de um estudo de abordagem descritivo e transversal, com direcionalidade temporal prospectiva. A pesquisa foi realizada em uma universidade privada no município de São Luís-MA, com amostra total de 451 estudantes universitários dos cursos de Enfermagem, Odontologia, Psicologia e Medicina. Para coleta de dados foram utilizados, Questionário de Dados Sociodemográficos e Escala de Avaliação do Risco de Suicídio de Columbia (C-SSRS). A pesquisa obedeceu aos aspectos éticos de acordo com a Resolução 466/12. Nos resultados, $80,3 \%$ eram do sexo feminino, 53,7\% com idade de 21 a 25 anos, 90,9\% não possuíam filhos e $43,0 \%$ eram católicos. De acordo com o C-SSRS, 39,5\% dos estudantes desejaram estar mortos, 29,3\% desejaram se matar e $12,4 \%$ pensaram em como tirar a própria vida. Portanto, a universidade precisa construir laços afetivos com seus alunos afim de melhorar seu prognóstico ainda no ambiente acadêmico. Dessa forma, o estudo chama atenção para a necessidade de construção e/ou melhorias de centros de acolhimento das demandas de saúde mental no âmbito das universidades para lidar com tal situação.

Palavras-chave: Estudantes; Saúde mental; Ideação suicida.

\begin{abstract}
Admission to the university represents a phase of change in social life and daily activities with autonomy and freedom for the student, and implies responsibilities in relation to academic charges. We realize that, as a result of these factors, many situations can make the student vulnerable to mental disorders. The objective of the study was to evaluate the mental health profile in relation to suicide risk among university students of a higher education institution. It is a descriptive and transversal approach study, with prospective temporal directionality. The research was conducted in a private university in the municipality of São Luís-MA, with a total sample of 451 university students from the Nursing, Dentistry, Psychology and Medicine courses. For data collection, a Sociodemographic Data Questionnaire and the Columbia Suicide Risk Assessment Scale (C-SSRS) were used. The research obeyed the ethical aspects according to Resolution 466/12. In the results, 80.3\% were female, 53.7\% were between 21 and 25 years old, $90.9 \%$ had no children and $43.0 \%$ were Catholic. According to C-SSRS, $39.5 \%$ of the students wanted to be dead, $29.3 \%$ wanted to kill themselves and $12.4 \%$ thought about how to take their own lives. Therefore, the university needs to build affective bonds with its students in order to improve its prognosis still in the academic environment. Thus, the study calls attention to the need to build and/or improve centers to meet the demands of mental health within universities to deal with such a situation.
\end{abstract}

Keywords: Students; Mental health; Suicidal ideation. 


\section{Resumen}

El ingreso a la universidad representa una fase de cambios en la interacción social y en las actividades diarias con autonomía y libertad para el estudiante, e implica responsabilidades frente a las demandas académicas. Nos dimos cuenta de que, como resultado de estos factores, muchas situaciones pueden hacer que el estudiante sea vulnerable a los trastornos mentales. El objetivo del estudio fue evaluar el perfil de salud mental en relación al riesgo suicida entre estudiantes universitarios de una institución de educación superior. Se trata de un estudio descriptivo y transversal, con direccionalidad temporal prospectiva. La investigación se realizó en una universidad privada de la ciudad de São Luís-MA, con una muestra total de 451 estudiantes universitarios de los cursos de Enfermería, Odontología, Psicología y Medicina. Para la recolección de datos se utilizó el Cuestionario de Datos Sociodemográficos y la Escala de Evaluación de Riesgo de Suicidio de Columbia (C-SSRS). La investigación siguió los aspectos éticos de acuerdo con la Resolución 466/12. En los resultados, el 80,3\% eran mujeres, el 53,7\% tenían entre 21 y 25 años, el 90,9\% no tenían hijos y el 43,0\% eran católicos. Según la C-SSRS, el 39,5\% de los estudiantes deseaba estar muerto, el 29,3\% deseaba suicidarse y el $12,4 \%$ pensaba en cómo quitarse la vida. Por tanto, la universidad necesita construir vínculos afectivos con sus estudiantes para mejorar su pronóstico incluso en el ámbito académico. Así, el estudio llama la atención sobre la necesidad de construir y / o mejorar centros de recepción de demandas de salud mental dentro de las universidades para hacer frente a esta situación.

Palabras clave: Estudiantes; Salud mental; Ideación suicida.

\section{Introdução}

O ingresso na universidade representa fase de mudanças no convívio social e nas atividades cotidianas, proporciona autonomia e liberdade ao estudante, mas também implica em responsabilidades frente às cobranças acadêmicas, independentemente de cor, gênero, idade ou meio cultural (Veloso et al., 2019). Para os autores, os estudantes universitários da área da saúde vivenciam diariamente dilemas específicos da profissão escolhida que envolve a dualidade entre saúde - doença e vida - morte.

Percebemos que, em decorrência desses fatores, muitas situações podem tornar o estudante vulnerável aos transtornos mentais, visto que, o ambiente acadêmico se torna uma competição, com cargas horárias excessivas e atividades curriculares e extracurriculares (Brondani et al., 2019).

Paralelamente, autores destacam que a distância da família tendo que morar muitas vezes em casas de parentes, ou casa alugadas ou casas universitárias possuindo assim confrontos com um conjunto de dificuldades, novas responsabilidades, incertezas, sentimentos de solidão, saudades de casa, da família e dos amigos são fatores preocupantes (Gonçalves; Freitas; Sequeira, 2011).

Nesse sentido, os discentes estão suscetíveis a diversos transtornos mentais, principalmente a depressão, Síndrome de Burnout, ansiedade, estresse, além de levar ao suicídio (Ariño; Bardagi, 2018). Esta situação agrava ainda mais, pois o estudante encara a morte como a única saída para as suas dificuldades ou problemas (Gonçalves; Freitas; Sequeira, 2011).

Nos últimos anos, a saúde mental do estudante universitário se tornou foco de atenção das literaturas. Pois, a área da saúde exige algumas características e expõe esses futuros profissionais a inúmeras situações de estresse que muitos não têm condições de enfrentar, como por exemplo as várias doenças e mortes, a pressão da sociedade, além dos grandes conteúdos o que faz com que esse indivíduo não tenha lazer (Lima et al., 2019).

Dentre todos os fatores estressantes que afetam essa população, observou-se que alguns eram fatores de risco especialmente para o suicídio, como transtornos de ansiedade e de humor, acidentes de trânsito, briga com agressão física, pouca confiança na comunicação com os pais, consumo de álcool e tabaco e abuso sexual (Siqueira et al., 2020).

De acordo com um estudo feito na Universidade de Salvador os sintomas de ansiedade e depressão entre alunos de medicina possuíram características superior à média encontrada na população em geral, ressalta-se a importância de destacar que o sofrimento causado ao discente tem sido um dos principais fatores para o suicídio no meio médico, com prevalência diretamente aos transtornos mentais (Neponuceno; Carvalho; Neves, 2019). Contudo, no Brasil, especificamente, estudos sobre 
essa temática encontram-se centralizados na região Sul, Sudeste e Centro-Oeste, locais em que são registradas maiores taxas de suicídios (Veloso et al., 2019).

No contexto epidemiológico brasileiro, os jovens na faixa etária dos 20 aos 24 anos, idade em que adentram as universidades e alcançam a vida profissional, em 2007 a taxa de suicídio alcançou 5,4 para cada 100.000 habitantes, o que representa um índice extremamente preocupante (Dutra, 2012).

Destacamos que, a ideação suicida em estudantes universitários se insere em um campo pouco explorado e marginalizado, o que demonstra a necessidade de desenvolvimento de pesquisas (Veloso et al., 2019). Pois, o suicídio, um ato voluntário e consumado por alguém que tem intenção de pôr fim à própria vida e que traga lesão à própria pessoa, independentemente do grau de letalidade, e a ideação suicida apresenta pensamentos como a intenção de colocar fim a própria vida (Vale, 2020).

Muitos estudos classificam o suicídio como um problema de saúde pública, mas apesar de seus altos índices nos dias, são dados subnotificados. Há uma relevância científica e social de se compreender o processo saúde-doença dessa população. Ressalta-se a importância de novos estudos que investiguem a relação dos aspectos específicos desta etapa de vida onde a literatura possui escassez e ainda se tem tabus. Dessa forma, o objetivo desta pesquisa foi avaliar o perfil de saúde mental com relação ao risco suicida entre estudantes universitários do ensino superior.

\section{Metodologia}

Estudo de abordagem descritivo e transversal com direcionalidade temporal prospectiva. A pesquisa foi realizada na Universidade Ceuma em 2 Campi - Anil e Renascença.

A população se constituiu de 451 estudantes universitários dos cursos de graduação de Enfermagem, Odontologia, Psicologia e Medicina. Foram incluídos estudantes universitários do primeiro ao último ano, maiores de 18 anos devidamente matriculados. E excluídos os que estavam de atestado médico, licença maternidade, não matriculados e menores de 18 anos.

Foram utilizados os seguintes instrumentos para coleta de dados: Questionário de dados sociodemográficos e Escala de Avaliação do Risco de Suicídio de Columbia (C-SSRS). Esta escala é utilizada em 45 países em 6 continentes. Consiste em perguntas simples cujo objetivo é avaliar se o entrevistado apresenta risco de cometer suicídio. Investiga se e quando o paciente teve ideação suicida, se realizou ações para se preparar para tal finalidade, caso tenha tentado suicídio, por qual motivo foi impedido e se, teve letalidade efetiva (Posner et al., 2011; CSSRS, 2016).

A Escala de Gravidade Suicida de Columbia foi desenvolvida na Universidade de Columbia-NY, pela equipe do Centro de Pesquisa de Risco de Suicídio, liderado pela Dra. Kelly Posner. É traduzida em mais de 100 línguas e vem sendo usada em recursos tanto de aplicação clínica, quanto de pesquisa. Embora exista uma tradução para o português brasileiro, ela ainda não possui validação para uso no Brasil, sendo este o objetivo da extensão deste projeto. Na escala quatro construtores são medidos, composto por perguntas organizadas em sub-escalas, relacionadas a: a) Ideação suicida, em que se avalia a presença desde pensamentos até ideações suicidas com planejamento ao longo da vida; b) Intensidade da ideação, na qual se quantifica a intensidade em uma escala de 1-5, abordando frequência, duração, controlabilidade, razões de causa e de impedimento; c) Comportamento suicida, avaliando a existência e características das tentativas de suicídio ao longo da vida; d) Escala de letalidade, que avalia tentativas reais (letalidade efetiva / danos físicos) classificada de forma ordinal com 5 pontos, e a letalidade potencial de tentativas que foi classificada de forma ordinal com 3 pontos (Posner et al., 2011). Neste estudo esta avaliação não foi realizada.

A coleta de dados seguiu todas as recomendações em razão dos casos de infecção por COVID-19. Primeiramente, se entrou em contato com os coordenadores dos cursos de graduação. Posteriormente, agendou-se com os líderes de turma do primeiro ao último ano, explicando a pesquisa, apresentando seus objetivos, com intuito de agendar as coletas de dados, que foi 
realizada de forma online através da plataforma Google Forms. Ao acessá-lo, o participante deparou-se com o Termo de Consentimento Livre e Esclarecido (TCLE), que ressaltava o caráter voluntário e sigiloso da pesquisa. Somente após concordar com o termo, o aluno poderia responder o questionário.

Para a análise dos dados obtidos, foi utilizado o software SPSS (versão 21.0) onde foram realizadas estatísticas descritivas para caracterização da amostra e para apresentar o perfil dos estudantes na C-SSRS. Em seguida, realizou-se testes de associação Qui-Quadrado de Pearson entre os grupos encontrados.

Esta pesquisa está ligada a um projeto maior, intitulado, "Saúde Mental Do Estudante Universitário: Fatores protetores e fatores de vulnerabilidade", que obedeceu ao posicionamento ético, norteou-se a partir das recomendações éticas dispostas nas Normas e Diretrizes que regulamentam a pesquisa envolvendo seres humanos, estabelecidas na Resolução 466/12 do Conselho Nacional de Saúde, sendo aprovada no Comitê de Ética em Pesquisa através do Parecer Consubstanciado $\mathrm{n}^{\circ}$ 4.127.772.

\section{Resultados e Discussão}

Os dados da pesquisa serão apresentados em forma de tabelas e discutidos conforme sua distribuição para melhor visualização e compreensão.

Tabela 1 - Distribuição dos dados sociodemográficos dos estudantes universitários. (N=451).

\begin{tabular}{|c|c|c|}
\hline Variáveis & $\mathrm{N}$ & $\%$ \\
\hline \multicolumn{3}{|l|}{$\begin{array}{l}\text { Sexo } \\
\end{array}$} \\
\hline Masculino & 88 & 19,5 \\
\hline Feminino & 362 & 80,3 \\
\hline Ausente & 1 & 0,2 \\
\hline \multicolumn{3}{|l|}{ Idades } \\
\hline Entre 18 e 20 anos & 140 & 31,0 \\
\hline Entre 21 e 25 anos & 242 & 53,7 \\
\hline Entre 26 e 30 anos & 40 & 8,9 \\
\hline Acima de 31 de anos & 29 & 6,4 \\
\hline \multicolumn{3}{|l|}{ Estado Civil } \\
\hline Solteiro(a) & 419 & 92,9 \\
\hline Casado(a) & 28 & 6,2 \\
\hline Divorciado(a) & 3 & 0,7 \\
\hline Separado(a) & 1 & 0,2 \\
\hline \multicolumn{3}{|l|}{ Filhos } \\
\hline Não tenho filhos & 410 & 90,9 \\
\hline Sim, um filho & 16 & 3,5 \\
\hline Sim, dois filhos & 16 & 3,5 \\
\hline Sim, três ou mais & 6 & 1,3 \\
\hline Ausente & 3 & 0,8 \\
\hline \multicolumn{3}{|l|}{ Moradia } \\
\hline Pais & 210 & 46,6 \\
\hline Familiares & 152 & 33,7 \\
\hline Amigos & 28 & 6,2 \\
\hline Sozinho(a) & 55 & 12,2 \\
\hline Ausente & 6 & 1,3 \\
\hline \multicolumn{3}{|l|}{ Religião } \\
\hline Católica & 194 & 43,0 \\
\hline Evangélica & 160 & 35,5 \\
\hline Espírita & 15 & 3,3 \\
\hline Ateu & 12 & 2,7 \\
\hline Outra & 68 & 15,1 \\
\hline Ausente & 2 & 0,4 \\
\hline
\end{tabular}




\begin{tabular}{lcc} 
Renda Familiar & & \\
Um salário mínimo & 62 & 13,7 \\
Dois salários mínimos & 103 & 22,8 \\
Três salários mínimos & 90 & 20,0 \\
Quatro salários mínimos & 56 & 12,4 \\
Cinco ou mais salários mínimos & 139 & 30,8 \\
Ausente & 1 & 0,3 \\
\hline
\end{tabular}

Fonte: Autores (2020).

Conforme observamos na Tabela 1, este estudo foi composto por 451 estudantes, dos quais $80,3 \%(n=362)$ eram do sexo feminino e 19,5\% ( $\mathrm{n}=88$ ) do sexo masculino. Indo de encontro aos achados de Brondani et al. (2019), onde estudos epidemiológicos mostram que o risco de desenvolver transtorno mental é três vezes maior em mulheres do que nos homens, e isto pode estar ligado ás oscilações hormonais.

Em relação à idade a maior prevalência era de 21 a 25 anos com 53,7\% (n=242); sobre o estado civil 92,9\% (n= 419) eram solteiros. Grande parte dos estudantes, relataram não ter filhos com 90,9\% $(n=410)$ e ainda morando com os pais, 46,6\% $(\mathrm{n}=210)$, ou com seus familiares, $33,7 \%(\mathrm{n}=152)$, e $12,2 \%(\mathrm{n}=55)$ sozinhos.

Cabe destacar que, em um estudo realizado pela Universidade Federal de Sergipe, revelou que a distância da família é um dos problemas desencadeadores da vontade de desistir da faculdade (Santos, 2018). A saída da casa de seus pais gera uma responsabilidade maior para esses estudantes, e isto pode estar ligado ao curso integral e as atividades domésticas. A distância do amor materno e paterno é um fator gerador de transtornos mentais que podem levar a ideação suicida. Esses jovens para se inserirem em um grupo que se sintam acolhidos muitas das vezes passam a experimentar bebidas e drogas ilícitas, por existe a liberdade já que estão longe dos seus pais (Figueira et al., 2020).

Sobre a religião, 43,0\% $(n=194)$ eram católicos, 35,5\% ( $n=160)$ evangélicos e 15,1\% ( $n=68)$ outros, que incluem o judaísmo e ubandismo. A religião se comporta como um fator protetor para a ideação suicida, pois contribui para o equilíbrio de emoções e sentimentos (Santos et al., 2017). A religião proporciona um ambiente interpessoal gerando uma ligação harmônica entre as pessoas melhorando assim, a qualidade de vida do indivíduo e diminuindo pensamentos suicidários, o fato do estudante ter alguma crença o ajuda para situações de desesperança, tristeza e dor.

Dos entrevistados 56,5\% ( $\mathrm{n}=255)$ eram de baixa renda. E, de acordo com Figueira et al. (2020), a classe social está diretamente ligada as doenças mentais devido aos frequentes sentimentos de humilhação e a diversos medo. Um estudo similar realizado com 714 estudantes na Universidade Federal de Mato Grosso (UFMT), relatou que os universitários de baixa renda apresentaram maior prevalência de ideação suicida ( $\mathrm{RP}=1,69)$ comparados com os de alta classe (Santos et al., 2017).

Tabela 2 - Caracterização da Escala de Avaliação do Risco de Suicídio de Columbia (C-SSRS). (N=451).

\begin{tabular}{lcc}
\hline Perguntas & $\mathrm{N}$ & $\%$ \\
\hline Você desejou estar morto/a ou desejou poder dormir e nunca mais & & 59,2 \\
acordar? & 267 & 39,5 \\
Não & 178 & 1,3 \\
Sim & 6 & 69,4 \\
Ausente & 313 & 29,3 \\
Você já pensou realmente em se matar? & 132 & 1,3 \\
Não & 6 & 84,0 \\
Sim & & 12,4 \\
Ausente & 379 & 3,6 \\
Você tem pensado em como poderia fazer isso? & 56 & 16
\end{tabular}




$\begin{array}{lcc}\text { Quantas vezes você teve esses pensamentos? } & \\ \text { Menos de uma vez por semana } & 123 & 27,3 \\ \text { Uma vez por semana } & 23 & 5,1 \\ \text { 2 - 5 vezes por semana } & 19 & 4,2 \\ \text { Todos os dias ou quase todos os dias } & 7 & 1,6 \\ \text { Muitas vezes ao dia } & 6 & 1,3 \\ \text { Ausente } & 273 & 60,5 \\ \text { Quando você tem esses pensamentos, quanto tempo eles duram? } & & \\ \text { Passageiros - alguns segundos ou minutos } & 107 & 23,7 \\ \text { Menos de 1 hora/ algum tempo } & 52 & 11,5 \\ \text { - 4 horas / muito tempo } & 23 & 5,1 \\ 4-8 \text { horas/ a maior parte do dia } & 9 & 2,0 \\ \text { Mais de 8 horas/ persistentes ou contínuos } & 4 & 0,9 \\ \text { Ausente } & 256 & 56,8 \\ \text { Você pôde / pode parar de pensar em se matar ou de querer } & & \\ \text { morrer se você quisesse / quiser? } & 111 & 24,6 \\ \text { É capaz de controlar os pensamentos facilmente } & 44 & 9,8 \\ \text { Pode controlar os pensamentos com pouca dificuldade } & 22 & 4,9 \\ \text { Pode controlar os pensamentos com alguma dificuldade } & 14 & 3,1 \\ \text { Pode controlar os pensamentos com muita dificuldade } & 13 & 2,9 \\ \text { Não tenta controlar os pensamentos } & 13 & 2,9 \\ \text { É incapaz de controlar seus pensamentos } & 234 & 51,8 \\ \text { Ausente } & \end{array}$

Há coisas - algo ou alguém que o/a impediram de querer morrer ou de colocar em ação sua ideia de cometer suicídio?

Essas razões, com certeza, o/a impediram.

Essas razões, provavelmente, o/a impediram.

Não tem certeza se essas razões o/a impediram.

Essas razões, provavelmente, não o/a impediram.

Essas razões, com certeza, não o/a impediram.

Não se aplica ao seu caso.

Ausente

Que tipos de razão você teve para pensar em querer morrer ou se matar? Foi para acabar com o sofrimento ou pôr fim à maneira como você estava se sentindo?

Com certeza para acabar com o sofrimento

Sobretudo para acabar com o sofrimento

Tanto para chamar atenção, se vingar ou provocar reação de outras

pessoas como acabar com o sofrimento.

Sobretudo para chamar atenção, se vingar ou provocar reação de outras pessoas.

Com certeza para chamar a atenção, se vingar ou provocar reação de outras pessoas.

Não se aplica no meu caso

Ausente

203

\section{Autolesão não suicida}

Não

Sim

Ausente

Houve alguma vez em que começou a fazer alguma coisa para pôr fim à sua vida, mas alguém ou alguma coisa o/a impediu antes que você realmente fizesse algo?

Não

Sim

Houve alguma vez em que você começou a fazer alguma coisa para

tentar pôr fim à sua vida, mas você mesmo/a parou antes de efetuar a ação?

Sim 


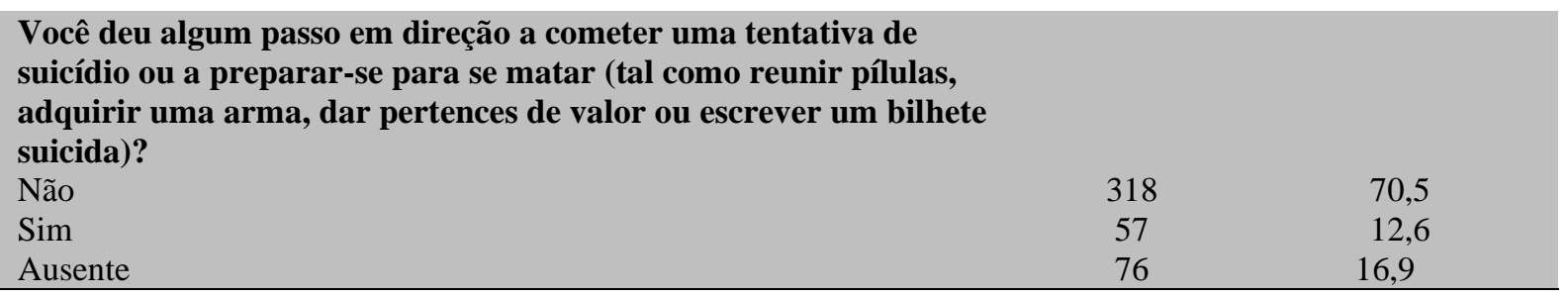

Fonte: Autores (2020).

De acordo com a Escala de Avaliação do Risco de Suicídio de Columbia (C-SSRS), como podemos verificar na Tabela 2. Observamos que 39,5\% ( $n=178)$ dos estudantes desejaram estar mortos; 29,3\% ( $n=132)$ desejaram se matar e 12,4\% $(\mathrm{n}=56)$ pensaram em como tirar a própria vida.

Ressaltamos que, o risco de suicídio se torna proporcional à ideação suicida, pois quanto maior o desejo da morte e o poder de letalidade do método de escolha, bem como o sentimento de tristeza, desesperança e o descontentamento com a vida, maiores são as chances do indivíduo desenvolver o comportamento suicida. Cerca de, 27,3\% ( $\mathrm{n}=123)$ desses estudantes relataram ter pensamentos suicidas menos de uma vez por semana; $17,8 \%(n=80)$ disseram ter algum tipo de dificuldade para controlar seus pensamentos suicidas, sendo elas pouco, alguma ou muito e 5,8\% ( $\mathrm{n}=26$ ) não tentam controlar seus pensamentos ou é incapaz de controla-los, isto nos mostra um fator preocupante, pois estes estudantes veem a morte como saída para seus sofrimentos, não tendo nenhum fator protetor para controlá-los.

Estes achados, dialogam com Santos (2018), que em seu estudo mostrou, que a fala dos universitários denotou uma desesperança em continuar vivendo, de acordo com um estudante, "Suicídio é uma escolha existencial. É o meio que encontramos para acabar com o sofrimento, é a angústia que corrói nossa existência".

Tabela 3 - Apresentação do Teste Qui-Quadrado entre os grupos.

\begin{tabular}{|c|c|c|c|c|}
\hline & $\begin{array}{c}\text { Ausência de } \\
\text { ideação suicida } \\
(n=222)\end{array}$ & $\begin{array}{l}\text { Ideação } \\
\text { suicida } \\
(n=73)\end{array}$ & $\begin{array}{l}\text { Comportamento } \\
\text { suicida } \\
(\mathrm{n}=57)\end{array}$ & p-valor \\
\hline \multicolumn{5}{|l|}{ Curso } \\
\hline Enfermagem & $86(64,7 \%)$ & $30(22,6 \%)$ & $17(12,8 \%)$ & 0,036 \\
\hline Medicina & $42(77,8 \%)$ & $8(13,0 \%)$ & $5(9,3 \%)$ & \\
\hline Odontologia & $34(65,4 \%)$ & $11(21,2 \%)$ & $7(13,5 \%)$ & \\
\hline Psicologia & $60(53,6 \%)$ & $24(21,4 \%)$ & $28(25,0 \%)$ & \\
\hline \multicolumn{5}{|l|}{ Religião } \\
\hline Católica & $89(58,9 \%)$ & $37(24,5 \%)$ & $25(16,6 \%)$ & 0,004 \\
\hline Evangélica & $92(77,3 \%)$ & $13(10,9 \%)$ & $14(11,8 \%)$ & \\
\hline Espírita & $6(54,5 \%)$ & $4(36,4 \%)$ & $1(9,1 \%)$ & \\
\hline Ateísmo & $5(45,5 \%)$ & $2(18,2 \%)$ & $4(36,4 \%)$ & \\
\hline Outras & $30(48,3 \%)$ & $17(29,3 \%)$ & $13(22,4 \%)$ & \\
\hline \multicolumn{5}{|c|}{ Apoio dos Colegas } \\
\hline Sim & $175(66,5 \%)$ & $50(19,0 \%)$ & $38(14,4 \%)$ & 0,056 \\
\hline Não & $47(52,3 \%)$ & $23(25,6 \%)$ & $19(22,1 \%)$ & \\
\hline \multicolumn{5}{|c|}{ Atividade física } \\
\hline Não prática & $85(52,8 \%)$ & $42(26,1 \%)$ & $34(21,1 \%)$ & 0,001 \\
\hline Pratica & $137(71,7 \%)$ & $31(16,2 \%)$ & $23(12,0 \%)$ & \\
\hline
\end{tabular}

Fonte: Autores (2020).

Observamos na Tabela 3, a apresentação do Teste Qui-Quadrado entre os grupos. E, a partir das respostas dos estudantes, os que responderam a todos os itens da escala puderam ser classificados em três grupos: 1) Ausência de ideação ou de comportamento suicida ( $\mathrm{n}=222)$; Ideação Suicida $(\mathrm{n}=73)$; e Comportamento Suicida $(\mathrm{n}=57)$. 
Conforme o teste Qui-Quadrado de Pearson o curso universitário associou-se significativamente aos grupos $(\mathrm{p}=$ 0,036), observou-se que o curso da enfermagem apresentou maior porcentagem, 22,6\% $(n=30)$ para ideação suicida seguido do curso de psicologia $21,4 \%(n=24)$ e de odontologia $21,2 \%(n=11)$.

A sobrecarga durante o curso é um fator preocupante, pois, isto pode gerar um estresse ao estudante contribuindo para a ideação suicida. Entretanto, um estudo realizado com estudantes de enfermagem em São Paulo revelou que estes apresentaram comportamento suicida principalmente por termos associados a sofrimento mental, desespero ou desesperança (Vedana; Zanetti, 2019). A diferença entre esses achados pode estar ligada aos diferentes tipos de instrumentos utilizados para caracterização da população, características singulares das regiões do estudo e a época em que esses estudantes foram estudados, ressaltando-se a importância de estudos mais profundos.

Com relação ao comportamento suicida o curso de psicologia obteve maior porcentagem, 25\% ( $n=28)$ seguido do curso de enfermagem com 12,8\% ( $n=17)$ e de odontologia com 13,5\% ( $n=7)$. Em contrapartida, um estudo feito em uma Instituição Pública de Ensino Superior do Piauí com estudantes de enfermagem, fisioterapia, medicina e psicologia revelou que os estudantes de psicologia apresentaram maior média para ideação suicida com relação aos outros cursos (Veloso et al., 2019).

De acordo com o Conselho Nacional de Saúde (CNS) (2018), na Resolução nº 597, de 13 de setembro de 2018, os cursos de psicologia no Brasil devem ter no mínimo 4.000 horas de curso nos 5 anos de forma presencial o que revela uma intensa atividade durante a graduação, exigindo dos alunos uma disponibilidade integral (CNS, 2018 apud Azevedo; Da Silva, 2019). Toda essa carga horária contribui para fatores suicidas, pois o estudante não possui momentos de lazer, tendo que se preocupar com as atividades da faculdade.

Destacamos que a religião, segundo Cardoso (2016), é considerada um fator protetor para os transtornos mentais, o indivíduo que possui crenças religiosas apresenta menor probabilidade de ter ideação suicida entre pessoas em risco. Neste estudo, esta variável se associou significamente aos grupos $(\mathrm{p}=0,004)$, observou-se maior ideação suicida entre os espíritas, com $36,4 \%(n=4)$ e um maior comportamento suicida entre os ateus, com $36,4 \%(n=4)$.

Cabe destacar que, o apoio dos colegas também se mostrou uma variável importante ( $p=0,056)$. Entre os que não sentem apoio dos colegas, 25,6\% ( $n=23)$ tiveram ideações suicidas e 22,1\% ( $n=19)$ teve comportamentos suicidas.

Em estudo desenvolvido por Santos et al. (2017), os vínculos interpessoais exercem forte influência no comportamento do indivíduo, pois durante a faculdade os alunos se tornam mais próximos visto que os cursos da área da saúde são em média cinco anos e de forma integral.

Em relação à prática de atividade física, apresentou associação com os grupos $(\mathrm{p}=0,001)$. Entre as pessoas que não praticam atividade física, 26,1\% ( $n=42)$ tiveram ideação e 21,1\% ( $n=34)$ já tiveram comportamentos suicidas.

Os fatores ambientais, como: alimentação saudável, atividade física, luz solar e ambiente construtivo para o lazer são indicativos de proteção para ideação e comportamentos suicidas (Gonçalves; De Freitas; Sequeira, 2011).

\section{Conclusão}

Podemos concluir neste estudo que, a prevalência dos estudantes universitários é do sexo feminino, que se encontram na faixa etária dos 21 a 25 anos, sendo a maioria solteiros, com grande parte sem filhos, e ainda morando com os pais. Muitos desses estudantes relataram ter o desejo da morte e como podem realizar tal ato.

Dessa forma, o presente estudo chama atenção para a necessidade de construção e/ou melhorias de centros de acolhimento das demandas de saúde mental no âmbito das universidades. Docentes e enfermeiros devem estar qualificados para lidar com tal situação afim de minimizar sua prevalência. $\mathrm{O}$ ambiente acadêmico deve preparar esses estudantes para lidar com a realidade fora do ambiente universitário. 
Vale ressaltar que a universidade precisa construir laços afetivos com seus alunos afim de melhorar seu prognóstico ainda no ambiente acadêmico, pois estes serão futuros profissionais e atenderão muitos pacientes com transtornos mentais, por isso precisam estar com uma boa saúde mental.

\section{Referências}

Ariño, D. O., \& Bardagi, M. P. Relação entre Fatores Acadêmicos e a Saúde Mental de Estudantes Universitários. Revista Psicologia em Pesquisa, 12(3), 2018.

Azevedo, A. K. S., Da Silva, M. V. M., \& da Silva Lima, A. P. Ideação e Tentativa De Suicídio Em Estudantes De Psicologia: Uma Dor Que Tem Morada Na Universidade. Holos, 6:1-13, 2019.

Brondani, M. A. et al. Depressão em estudantes universitários: fatores de risco e protetivos e sua relação nesse contexto. Disciplinarum Scientia| Saúde, 20(1): 137-149, 2019.

Cardoso, G. T. Comportamtentos autolesivos e ideação suicida nos jovens. 2016. 66 f. Dissertação (Mestrado Integrado em Medicina). Faculdade de Medicina da Universidade de Coimbra, Portugal, 2016.

CNS. Conselho Nacional de Saúde. 2018. Resolução Nº 597, de 13 de Setembro de 2018. Brasília. <http://conselho.saude.gov.br/resolucoes/reso_18.htm>.

CSSRS. The Columbia Lighthouse Project: Identify Risk, Prevent Suicide. 2016. < https://cssrs.columbia.edu/the-columbia-scale-c-ssrs/cssrs-for-research/ >.

Dutra, E. Suicídio de universitários: o vazio existencial de jovens na contemporaneidade. Estudos e Pesquisas em Psicologia, 12(3): 924-937, 2012.

Figueira, G. M., Demarchi, M. E., Casselli, D. D. N., De Sousa Martins, E., \& Souza, J. C. Fatores de risco associados ao desenvolvimento de transtornos mentais em estudantes universitários. Research, Society and Development, 9(9), e432997454-e432997454.

Gonçalves, A. M., De Freitas, P. P. \& Sequeira, C. Comportamentos suicidários em estudantes do ensino superior: factores de risco e de proteção. Millenium, 40: 149-159, 2011.

Lima, S. O. et al. Prevalencia de la Depresión en la Académicos del Área de la Salud. Psicologia: Ciência e Profissão, $39,2019$.

Neponuceno, H. D. J., Carvalho, B. D. N. D., \& Neves, N. M. B. C. Trastornos mentales comunes en estudiantes de medicina. Revista Bioética, 27(3):465$470,2019$.

Posner, K. et al. The Columbia-Suicide Severity Rating Scale: initial validity and internal consistency findings from three multisite studies with adolescents and adults. American journal of psychiatry, 168 (12): 1266-1277, 2011.

Santos, H. G. B. D. et al. Fatores associados à presença de ideação suicida entre universitários. Revista Latino-Americana de Enfermagem, $25,2017$.

Santos, E. K. T. D. A saúde mental dos universitários de terapia ocupacional: a cobrança do conhecimento técnico e científico frente a sua formação acadêmica. 2018. 34 f. Trabalho de Conclusão de Curso - Departamento de Terapia Ocupacional, Universidade Federal de Sergipe, Sergipe, 2018.

Siqueira, A. F. et al. Validação de manual sobre prevenção do suicídio para universitários: falar é a melhor solução. Revista Rene, 2020.

Vale, A. C. D. C. Ansiedade social e ideação e comportamento suicida em estudantes universitários. 2020. Dissertação (Mestrado em Psicologia Clínica e da Saúde) Faculdade de Folisofia e Ciências Sociais, 2020.

Veloso, L. U. P. et al. Ideação suicida em universitários da área da saúde: prevalência e fatores associados. Revista Gaúcha de Enfermagem, $40,2019$.

Vedana, K. G. G, \& Zanetti, A. C. G. Atitudes de estudantes de enfermagem relacionadas ao comportamento suicida. Revista Latino-Americana de Enfermagem, 27, 2019. 